\title{
MODELO TEÓRICO Y MÉTODO PARA LA DETERMINACIÓN DE LOS FACTORES CLAVE DE LA EFECTIVIDAD DE LA DIRECCIÓN DE PROYECTOS DE VIVIENDA RURAL EN LOJA ECUADOR
}

\author{
Model theory and method for the determination of the key factors in the \\ effectiveness of the projects management of rural housing in Loja Ecuador
}

EPISTEMUS

ISSN: 2007-8196 (electrónico)

ISSN: 2007-4530 (impresa)

Servio Tulio Burneo Valarezo 1

Salvador Felipe Espinet Vázquez ${ }^{2}$

Recibido: 14 de octubre de 2018 ,

Aceptado: 13 de noviembre de 2018

Autor de Correspondencia:

MBA Servio Tulio Burneo Valarezo

Correo: servioburneo@hotmail.com

\section{Resumen}

Los intentos de elaborar una metodología general aplicable a todo tipo de proyecto en cualquier entorno han fracasado, pues a mayor generalización se vuelven más abstractas e inoperantes. Optimizar los recursos puestos a disposición del proyecto por las partes interesadas, evitando que por falta de previsión, gestión y control, se desvíen, derrochen o se malversen recursos, ocurre con frecuencia sobre todo en obras públicas en programas de gobierno.

El objetivo de la investigación es presentar una propuesta de modelo teórico y un método con enfoque sistémico que nos ayuden a determinar los indicadores claves de efectividad en sus fases de desarrollo para el éxito de los proyectos de los programas de vivienda rural en la Provincia de Loja, Ecuador, adecuando los procedimientos del PMBOK del PMI, a las condiciones específicas del entorno, detallando las variables políticas, jurídicas, culturales y perceptuales en que se desarrolla dicho programa, poniendo al alcance de las partes interesadas la información integral y oportuna para la toma de decisiones, de forma que dicho programa contribuya al desarrollo rural sustentable.

Palabras clave: modelo teórico, método, enfoque sistémico, indicadores clave, sustentabilidad, proyectos de vivienda rural.

\section{Abstract}

Attempts to develop a general methodology applicable to all types of project in any environment have failed, since greater generalization become more abstract and inoperative. Optimize the resources available to the project by stakeholders, avoiding that for lack of foresight, management and control, deviate, squandering or be misused resources, frequently occurs mostly in public works in government programs.

The research aims to present a proposal for a theoretical model and a method with systemic approach that will help us to determine the key indicators of effectiveness in their stages of development to the success of the projects of rural housing programs in the Province of Loja, Ecuador, adapting the procedures of the $P M I P M B O K$, to the specific conditions of the environment, detailing the political, legal and cultural variables and perceptual in which this program develops, making parts available stakeholders information comprehensive and timely for the decision-making process, so that this programme will contribute to sustainable rural development.

Keywords: model theory, method, systemic approach, key indicators, sustainability, rural housing projects

1 Profesor ocasional de la Escuela Politécnica Nacional del Ecuador. servioburneo@hotmail.com

2 Profesor del Instituto Tecnológico de Chetumal. sfespinet@gmail.com 


\section{INTRODUCCIÓN}

La Provincia de Loja, al sur del Ecuador, se encuentra alejada de los centros de podery desarrollo, ha manteniendo tasas altas de analfabetismo, pobreza y migración del país por muchos años. En la actualidad el regreso de migrantes ecuatorianos, el desarrollo del turismo rural, son factores que han dado el incentivo económico que hacía falta, lo cual se ha ido complementando con proyectos de programas de vivienda rural.

La investigación presenta una propuesta de modelo teórico y un método para determinar los indicadores claves que deben tenerse en cuenta para lograr la efectividad de la Dirección de Proyectos en las fases de Diseño y Construcción lo que permitirá a las empresas y organizaciones locales involucradas en el programa de la vivienda rural de la Provincia de Loja en Ecuador, mejorar su accionar siguiendo el proceso del desarrollo de los proyectos con enfoque sistémico; mejorar el control y la toma de decisiones, lograr eficacia y eficiencia, optimizar recursos que en definitiva ayudará al éxito del programa de la vivienda rural para el desarrollo sustentable sostenible del Sur del Ecuador.

La falta de enfoque sistémico en la concepción y desarrollo de los proyectos de programas de vivienda, la falta de procedimientos o la aplicación de procedimientos inadecuados, para la previsión, gestión y control de los recursos, provocan frecuentemente el desvío, derroche o su malversación con el resultado en el fracaso por la no sustentabilidad de los proyectos.

La singularidad de los proyectos radica en que se desarrollan en un entorno físico y temporal específico. Su configuración y alcance, que en el caso de proyectos de construcción de viviendas rurales se refiere a la tipología de la obra, puede ser repetitiva o típica pero el resto de los objetivos como es el plazo, costo y la calidad depende de la ubicación y otros factores o variables políticas, económicas, jurídicas, culturales o sociales, ambientales y sicoperceptuales relacionadas con las partes interesadas de cada proyecto específico, como pueden ser el promotor, patrocinador o financista, propietario o usuario, diseñadores, constructores, proveedores, etc..

La actividad profesional de "Dirección de Proyecto" en las fases de definición y ejecución, es la organización, supervisión y control de las operaciones necesarias para la materialización del proyecto (García, 2006). Lamentablemente las empresas e instituciones que participan en el programa de viviendas rurales de la Provincia de Loja, Ecuador, no cuentan con un sistema de Dirección por Proyectos formalizado.

Por este motivo las dificultades que encuentran comúnmente, para llevar a cabo la "Dirección de Proyecto en las Fases de su Desarrollo" efectiva, es motivo de investigación en el presente estudio. La falta de un modelo teórico (y un método) con enfoque sistémico, para determinar los indicadores críticos para la efectividad de los procedimientos de dirección de proyectos de viviendas

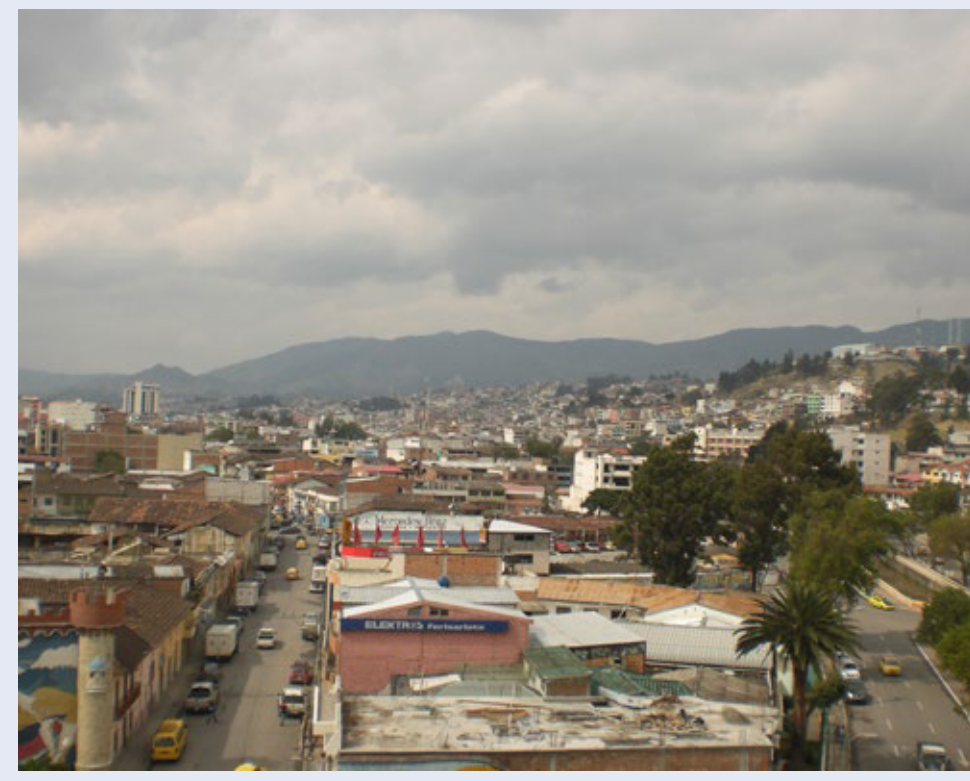

rurales, es lo que dificulta la estimación y control del tiempo en la ejecución de los tiempo, costos, especificaciones de calidad, gestión de servicios, reclamos y quejas, procesos a seguir y logro de la satisfacción de clientes o usuarios.

Las ventajas de contar con un Sistema de Dirección para las fases de desarrollo de los proyectos del programa de viviendas rurales de la Provincia de Loja en Ecuador se sintetizan en lo siguiente:

* Mayor control de los riesgos de los proyectos.

* Mejora la unión y el desarrollo del equipo de proyecto. Al obtener resultados positivos se puede convencer a los reacios al cambio y animar al resto para aprender más y seguir buscando efectividad.

* Aumento de calidad y cantidad. Al tener mayor eficiencia, les permite a las empresas y organizaciones involucradas a optimizar recursos y lanzar nuevos servicios de un nivel mayor, con una posición privilegiada.

* Nuevas oportunidades. Al tener un control en los procesos gracias al sistema de dirección para las fases del desarrollo del proyecto, se puede ofrecer la oportunidad de expansión, subcontratando y delegando, ya que se sabe mucho más de las partes del proyecto que no se desarrollan directamente y se pueden ofrecer nuevos servicios.

* Eficiente entrega de proyectos. Formalizar métricas de desempeño, obteniendo así procesos de mejora, proporcionando a todos los involucrados que puedan seguir con facilidad el proyecto en alcance, tiempo, costo y calidad como se había fijado, agilizando el proceso de entrega a clientes o usuarios.

* Aumentar la satisfacción de los clientes o usuarios. Un cliente agradecido, se convierte en un nuevo socio, ya que avala el desempeño de las empresas que acometieron el proyecto. 


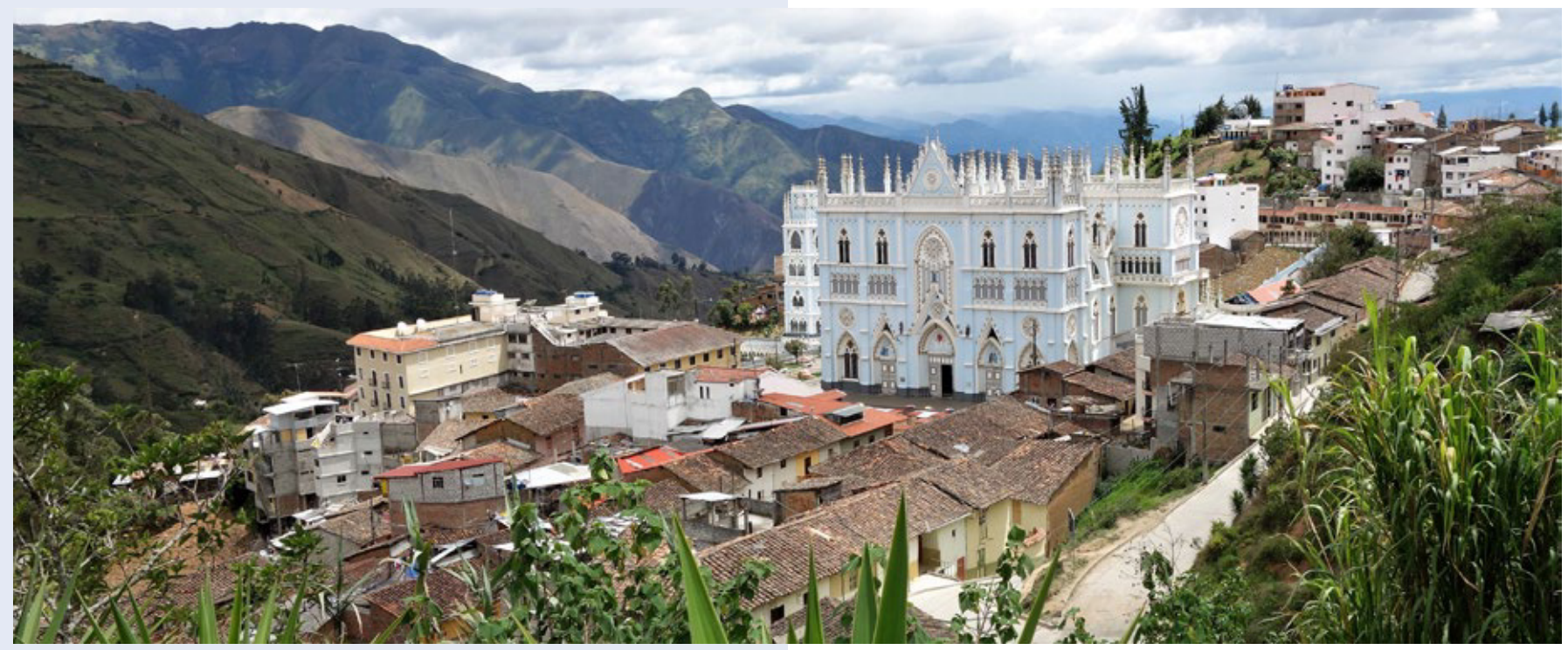

* Mayor ventaja competitiva. La mejor relación con los clientes o usuarios, es una fortaleza para la propagación de la difusión y así poder contar con un mejor posicionamiento y reputación en el mercado.

El análisis de la situación problemática es equivalente a identificar la necesidad que origina el problema según lo plantean (Espinet \& Sánchez, 2017), así mismo, estos autores indican que para esto se debe de aplicar técnicas de recolección de datos, o bien la gestión de información, a través de entrevistas o encuestas a las partes interesadas, o búsqueda de información en documentos impresos o digitales, aplicando métodos y técnicas de la investigación cualitativa.

Un diagnóstico de la situación de los resultados del programa de viviendas rurales en la Provincia Loja en Ecuador, antes del inicio de la investigación, aplicando los métodos y técnicas de la investigación cualitativa fundamentó la necesidad de la investigación y la delimitación del problema.

Por este motivo, surge la necesidad de encontrar una solución a la problemática de las empresas y organizaciones involucradas en el programa objeto de estudio de viviendas rurales en la Provincia de Loja Ecuador, que no cuentan con procesos formalizados e integrales para la "Dirección de Proyectos" para las fases diseño y ejecución. La falta de definición y determinación de los indicadores claves de la efectividad, provocan grandes fugas de recursos, tanto humanos, de tiempo y financieros, dejando insatisfechos a los clientes o beneficiarios del programa.

Diseñar un modelo teórico y un método, permite definir y determinar los factores claves de efectividad a tener en cuenta para para la Dirección de Proyectos adecuando al entorno local específico de cada proyecto los procedimientos del $\mathrm{PMBOK}^{\circledR}$ del $\mathrm{PMI}{ }^{\circledR}$. Vale mencionar que dichos procedimientos se utilizan en un sin número de empresas y organizaciones en otros entornos con gran éxito, no así en las fases de diseño y construcción del programa de viviendas rurales en la Provincia de Loja, en Ecuador.

El problema científico radica entonces en generar el conocimiento que permita responder la pregunta: ¿Cuál será el modelo teórico (y el método) que permita determinar los factores claves en la efectividad de los procedimientos de Dirección de Proyecto (Project Management) para las fases de diseño y construcción de proyectos del programa de vivienda rural en la Provincia de Loja, Ecuador, que contribuyan al desarrollo rural sustentable?

El objetivo general de la investigación es: Proponer un modelo teórico y un método que permita determinar los factores claves en la efectividad de los procedimientos de Dirección de Proyecto para las fases de diseño y construcción de proyectos del programa de vivienda rural en la Provincia de Loja, Ecuador, que contribuyan al desarrollo sustentable y sostenible.

El no haber podido encontrar en la amplia bibliografía consultada, referencias a modelos teóricos (y métodos) similares con igual propósito, supone que los resultados previstos de la investigación aportan a la generación de conocimientos con valor científico y metodológico.

El principal aporte se considera es el valor metodológico de la propuesta. Aunque se ha tomado como caso de estudio el Programa de la Vivienda Rural en la Provincia de Loja Ecuador y como corresponde a resultados de investigación cualitativa, estos no pueden generalizarse, la metodología ha sido suficientemente fundamentada para servir de referencia para la adecuación a otros entornos y programas de proyectos, lo que significaría una contribución al desarrollo de la disciplina de la Dirección de Proyectos y a la formación de profesionales de carreras afines a la construcción.

El paradigma de la investigación cualitativa de la episteme pos positivista ha tenido un amplio desarrollo en las Ciencias Sociales y más recientemente en Ciencias de la Salud, sin embargo no se encuentran suficientes 
desarrollos en las Ciencias Técnicas en especial en Ingeniería Civil y Arquitectura, por lo que este estudio puede ser una modesta contribución a la necesaria difusión de esta episteme en dichas áreas.

\section{DESCRIPCIÓN DEL MÉTODO}

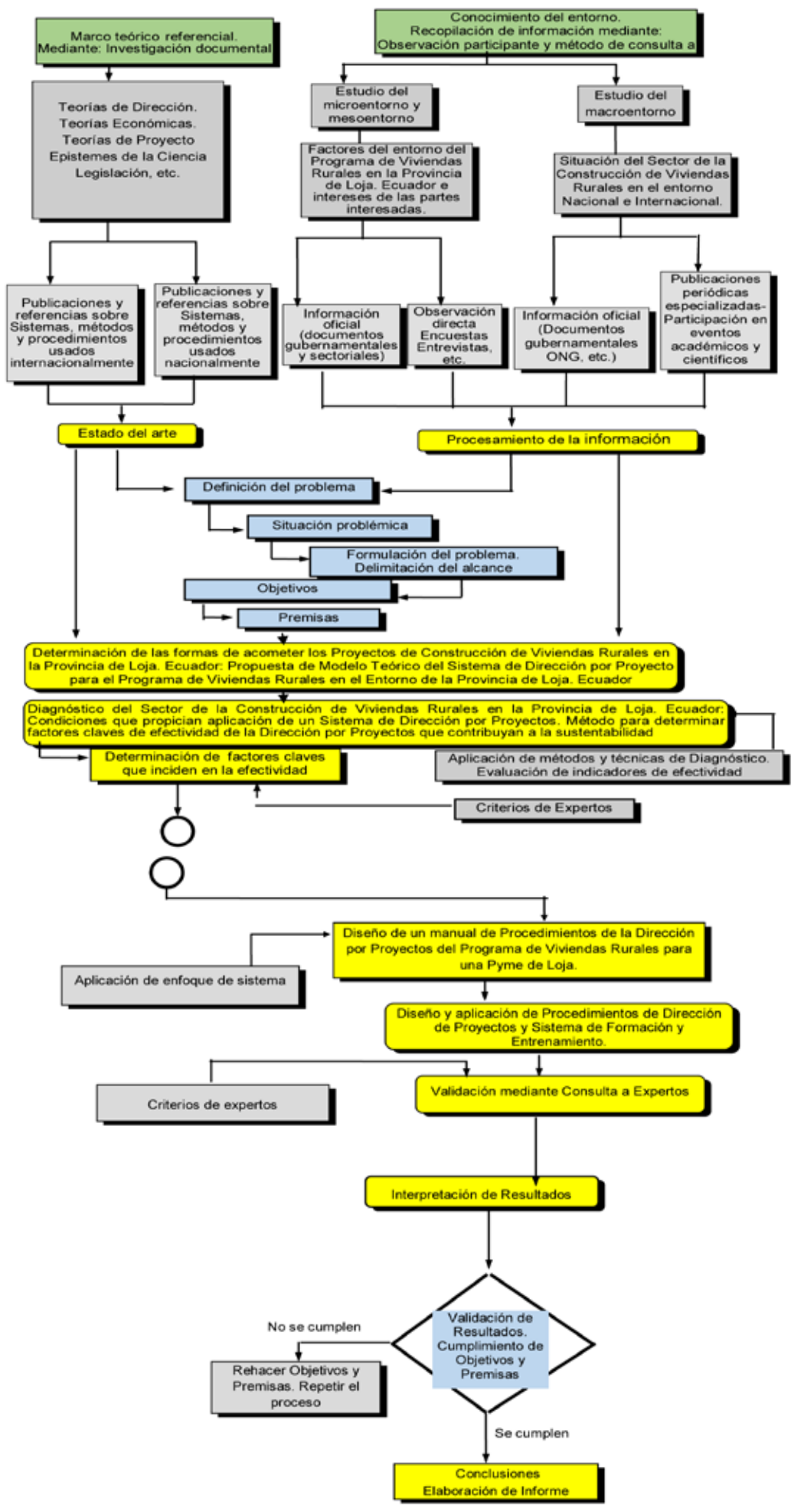

Figura 1. Diagrama de Flujo General de la Investigación.

Fuente: Elaboración propia. 2018

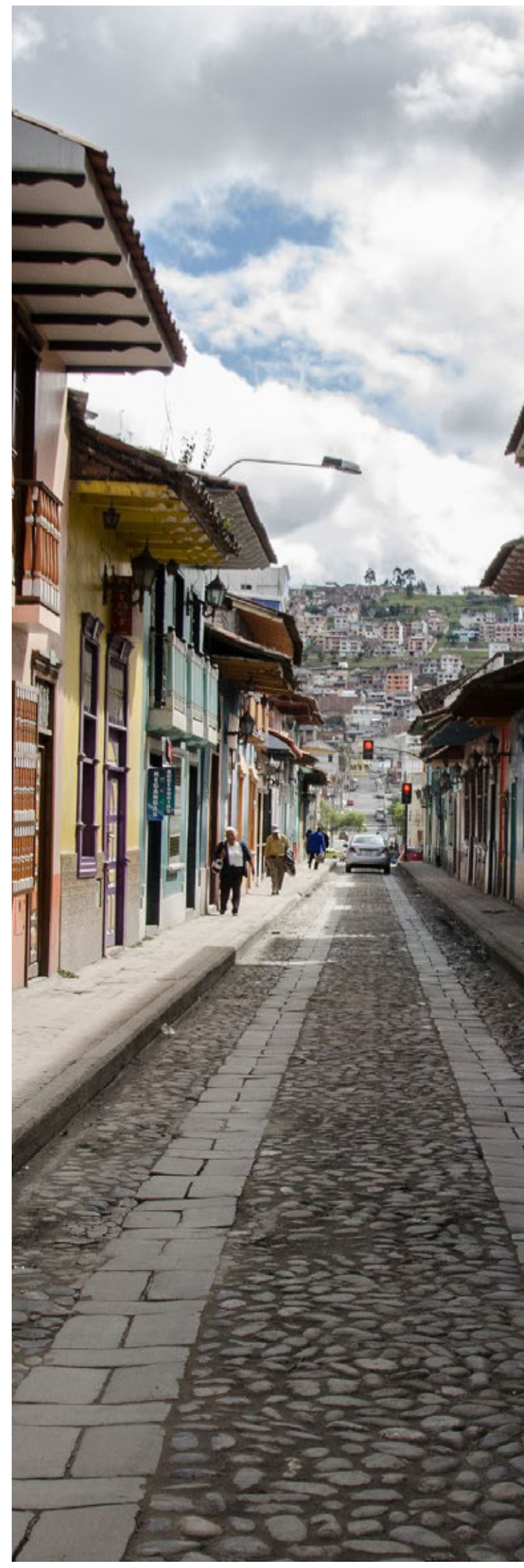




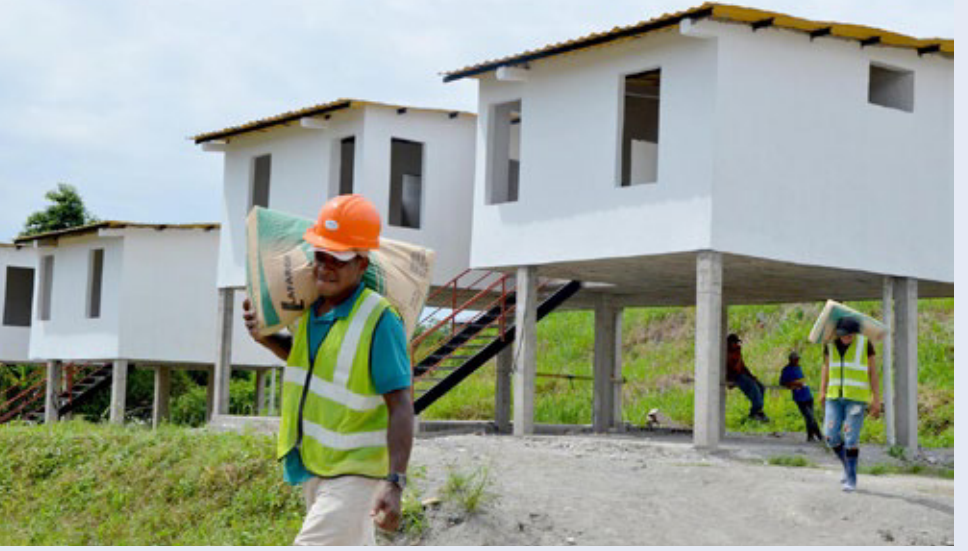

El método general propuesto por los autores, considera una metodología para la solución de problemas de proyecto como problemas de investigación, aplicando los epistemes pospositivistas del paradigma de la investigación cualitativa.

El modelo teórico resultante tiene ante todo, valor metodológico. Puede servir de referencia para otros proyectos. El esquema metodológico para la aplicación de este método se muestra en la Figura 1 y consta de varios pasos, la secuencia de tareas con los métodos y técnicas de investigación se lo explica a continuación:

* Estudio del estado del arte: Elaboración del Marco Teórico referencial sobre la teoría de dirección y otras teorías de la episteme pospositivista de la ciencia, asociadas al tema.

* Conocimientodelentorno:Recopilacióndeinformación sobre la situación del Sector de la Construcción de la Vivienda Rural en la Provincia de Loja. Ecuador y a nivel internacional, a través de encuestas, entrevistas, estudios de casos, investigaciones precedentes, análisis de la experiencia acumulada, participación en eventos académicos y científicos, etc.

* Evaluación de las formas de acometer los Proyectos de Construcción del Programa de Viviendas Rurales en la Provincia de Loja. Ecuador: Valoración de ventajas y desventajas, lo que unido al resultado de los pasos anteriores permite seleccionar qué método y técnica aplicar en las condiciones del entorno local.

* Diagnóstico de la situación del sistema Sector de la Construcción del Programa de la Vivienda Rural en la Provincia de Loja, para la aplicación de la propuesta de un sistema de dirección por proyecto: Aplicación de diferentes técnicas para el estudio del sistema (Estructura organizativa) y su relación con el entorno, mediante técnicas específicas de recogida de información como entrevistas, encuestas y sondeos de opinión, análisis de contenido, técnicas de trabajo creativo en grupos de expertos como "brainstorming" y otros métodos intuitivos y exploratorios de la prospectiva.
* Determinación de los factores claves para la aplicación de la técnica a implementar y la percepción que sobre estos factores tiene la alta dirección de la Pyme seleccionada como caso de estudio del sistema empresarial de la construcción de viviendas rurales en el territorio

* Diseño y aplicación de Procedimientos para la implementación del método: Lo que equivale al "know how" para la aplicación y la esencia de la transferencia tecnológica pues implica adecuarla a las condiciones locales. Se realiza mediante un proceso interactivo de debate y ajuste con los protagonistas de su aplicación y con los criterios de expertos consultados.

* Control de aplicación y Evaluación de resultados: Definición de las variables e indicadores y métodos que permiten validar los Procedimientos, evaluar los resultados de los Proyectos, las ventajas de la aplicación de la Dirección de Proyectos (DIP) y corroborar el logro de objetivos y cumplimiento de premisas.

* Interpretación de resultados: Corroborar la validez de la propuesta de modelo teórico y método para la determinación de los factores claves que inciden en la efectividad de la dirección de proyectos de construcción de viviendas rurales en la Provincia de Loja. Ecuador y contribuyen a la sustentabilidad de dicho programa. Se valora, teniendo en cuenta los postulados de la Teoría de Sistema, las posibilidades de extender y generalizar la experiencia con las adecuaciones necesarias a los requerimientos del entorno especifico. $\mathrm{Si}$ esta comprobación arroja que los resultados no se corresponden con lo esperado, no se alcanzan los objetivos o no se cumplen las premisas, entonces el problema no ha sido resuelto o no hay fundamento científico que sustente la propuesta, en cuyo caso se revisa si los métodos y técnicas fueron utilizados correctamente, se reformula el alcance, los objetivos y premisas y se repite el proceso investigativo.

* Conclusiones: Cuando los resultados obtenidos permitan considerar que se alcanzan los objetivos y se cumplen las premisas, se redactan las conclusiones del trabajo investigativo, así como las recomendaciones necesarias.

\section{RESULTADOS Y DISCUSIÓN}

Con los resultados obtenidos, se procede a representar el Modelo Teórico del Sistema Dirección por Proyecto y su Entorno para las empresas que brindan servicios de Dirección de Construcciones en el Programa de la Vivienda Rural en la Provincia de Loja. Ecuador. El modelo resultante se muestra en la Figura 2.

Las variables del entorno con mayor incidencia (Muy Fuerte), consideradas por los Expertos fueron la Económica y la Técnico Funcional; luego con menor incidencia (Fuerte a Media) le siguen la variable Política, Jurídica, Ambienta y la Histórica Cultural. 


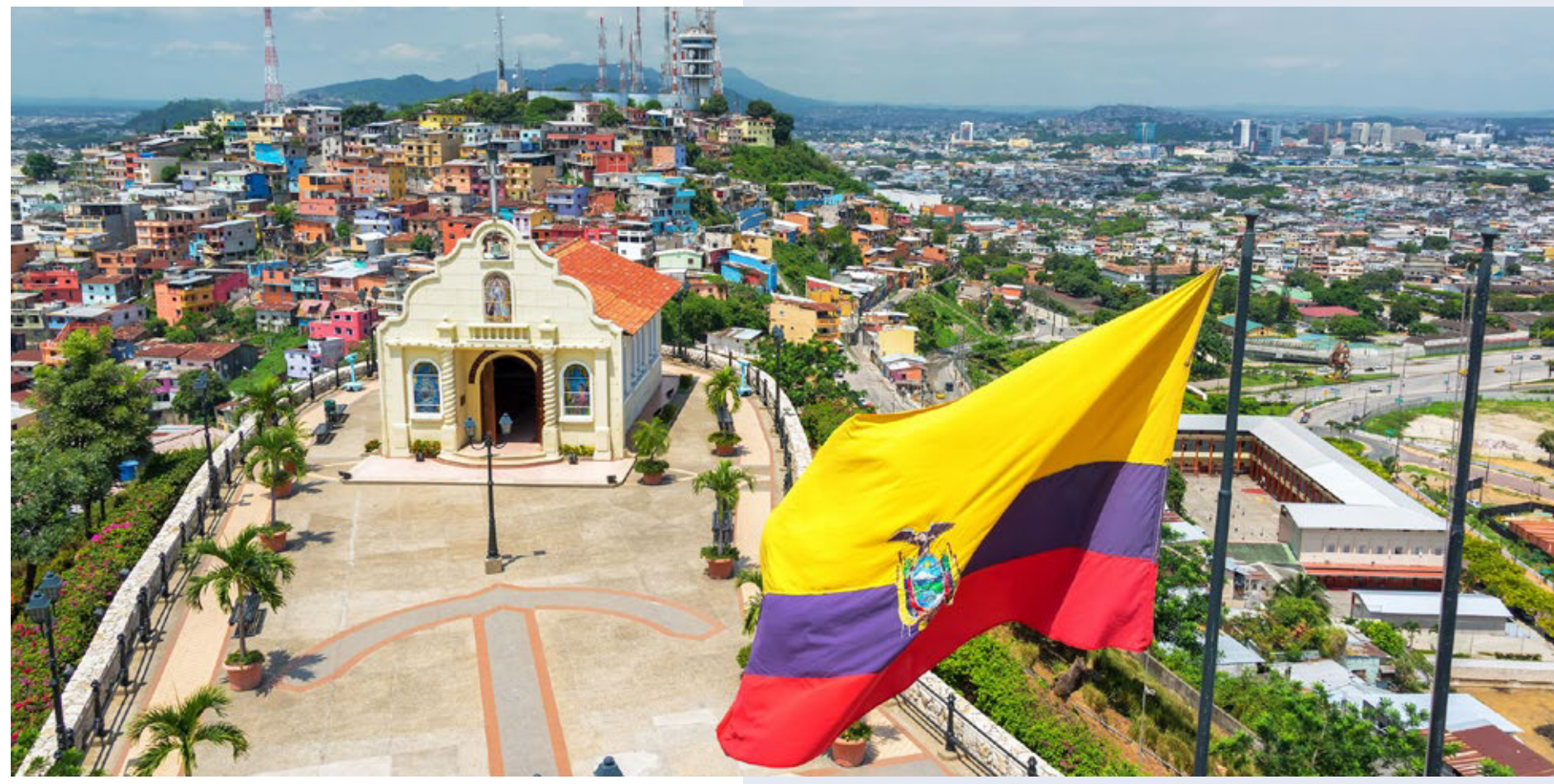

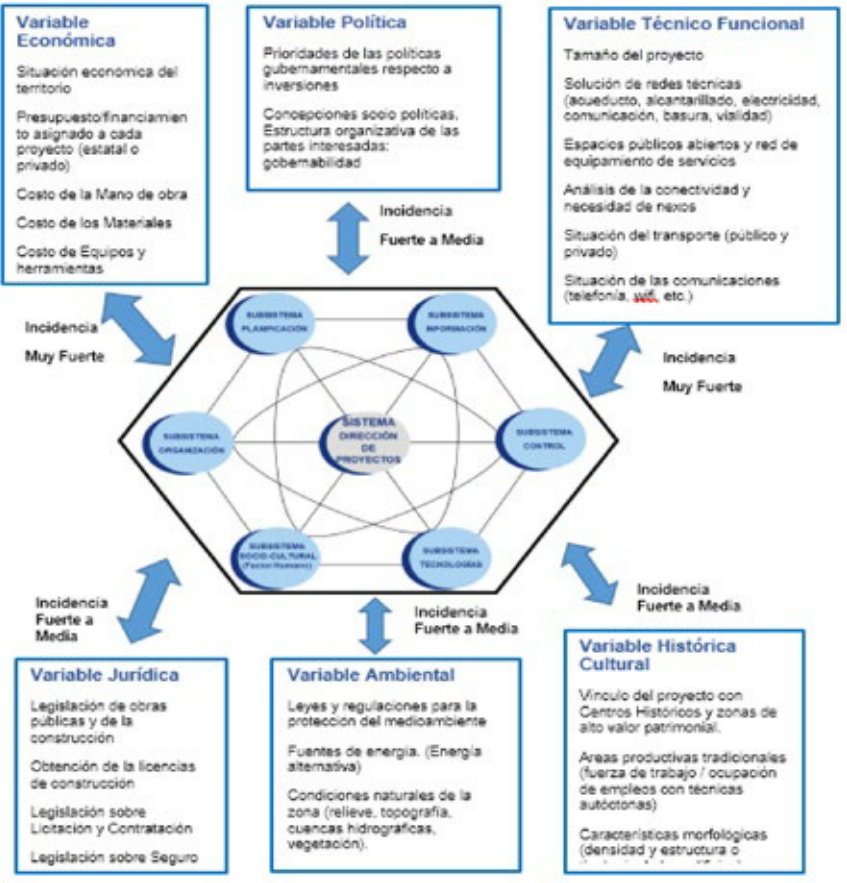

Figura 2. Modelo Teórico del Sistema Dirección por Proyecto y su Entorno para las empresas que brindan servicios de Construcciones en el Programa de la Vivienda Rural en la Provincia de Loja Ecuador.

Fuente: Elaboración propia (2017)

Según los Expertos consultados, los subsistemas o variables internas con mayor incidencia en el Sistema de Dirección por Proyecto, para las empresas que brindan servicios de Construcciones en el Programa de la Vivienda Rural en la Provincia de Loja. Ecuador, son el de Planificación, Información y Control, que son factores claves para la efectividad de la Dirección de Proyectos y además se requiere diseñar procedimientos que garanticen su integración, como se muestra en la Figura 3.

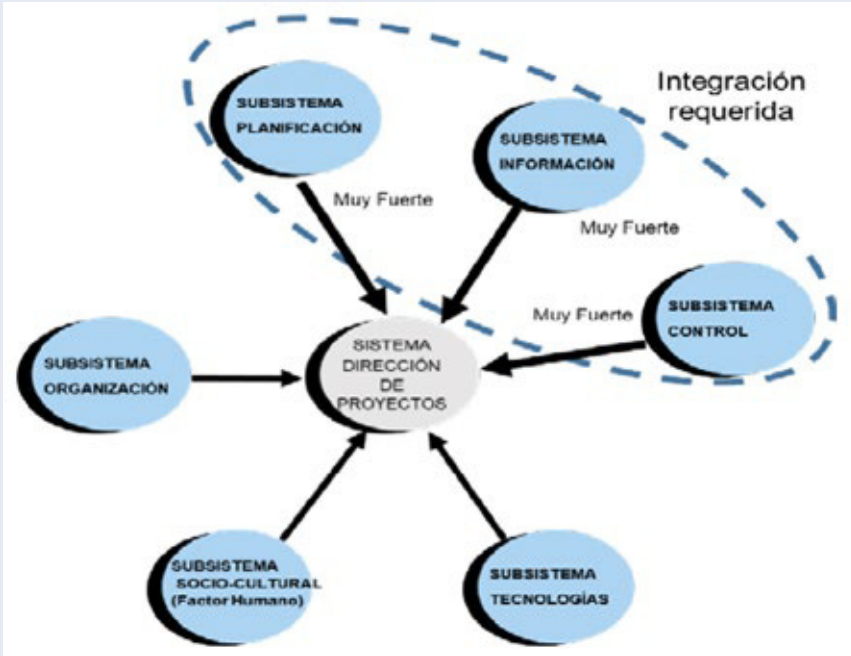

Figura 3. Subsistemas con mayor incidencia en el Sistema de Dirección por Proyecto y requerimiento de integración.

Fuente: Elaboración propia (2017).

Podemos desagregar los procesos y tareas de cada subsistema de la Dirección por Proyectos; por ejemplo para el Subsistema Planificación tal como se muestra en la Figura 4. 


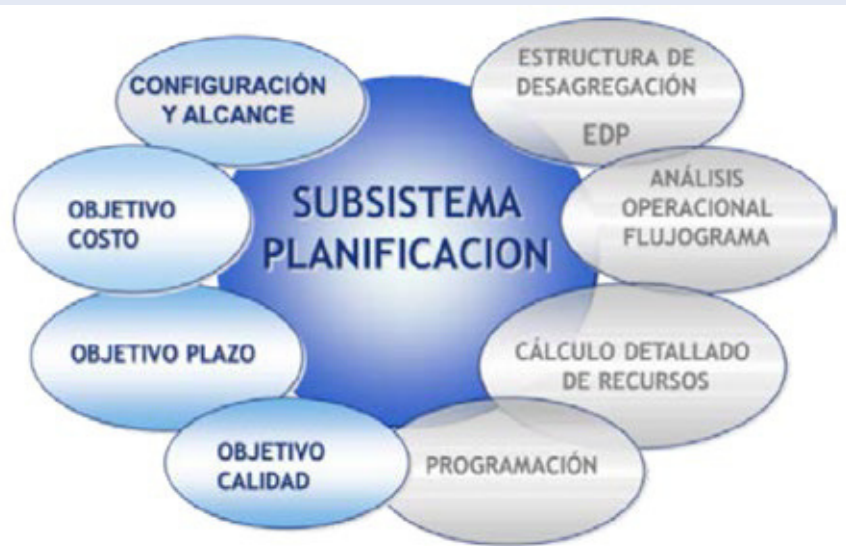

Figura 4. Desagregación de procesos del Subsistema

Planificación de la Dirección de la Empresa que

brinda servicios de Dirección de Construcciones en la

Provincia de Loja. Ecuador. Fuente: Adaptado del original de Ciencia del Proyecto.

Espinet y Sánchez (2017).

\section{CONCLUSIONES}

Tomando como base la demanda específica la vivienda rural en la Provincia de Loja, el reto de la presente investigación será diseñar empresas locales de vivienda eficaces y eficientes, que optimicen los recursos, con responsabilidad y principios de sostenibilidad, con enfoque sistémico, como lo determina el Project Management, ya aplicados exitosamente en el mundo por grandes empresas en proyectos de inversión.

Se asume para el desarrollo de la investigación el paradigma pos positivista y para la propuesta de modelo teórico y método, un enfoque sistémico y la metodología de la investigación cualitativa y con el método de consulta a expertos, determinar con rigor científico y suficiente certeza, los factores o variables claves del entorno de las Pymes vinculadas al Programa de Viviendas Rurales, con mayor incidencia en la efectividad de la dirección de sus proyectos.

En la mayoría de las Pymes vinculadas al programa de viviendas rurales, la deficiente definición del alcance del proyecto, de los objetivos básicos de plazo, costo y calidad y una pobre gestión de riesgos, son las tres principales causas por las cuales los proyectos no tienen el éxito esperado.

En el entorno de las empresas que brindan servicios de construcción en Loja. Ecuador, para el Programa de Viviendas Rurales, las variables o subsistemas de dicho entorno que más inciden en la dirección de sus proyectos son la variable Económica y la variable Técnico- funcional con incidencia fuerte; la variable Jurídica y la Política con incidencia de fuerte a media; con poca incidencia están las variables Medioambiental e Histórico Cultural y finalmente con débil o ninguna incidencia las Sociológica y Psicoperceptual.

\section{BIBILIOGRAFÍA}

[1] Amendola, L. (2004), Estratégicas y Tácticas en Dirección y Gestión de Proyectos. Project Management. Universidad Politécnica de Valencia.

[2] BUENO, E. (2007), Organización de Empresas. Estructura, procesos y modelos, 2. ${ }^{\mathrm{a}}$ ed., Madrid: Pirámide.

[3] Burneo, S., Delgado R, (2011), La Gestión Empresarial para la construcción de viviendas de interés social, Caso de Estudio la Provincia de Loja-Ecuador. Ponencia, III Congreso RIIPRO, Hermosillo México.

[4] Burneo, S., Delgado R, (2014), La Gestión Empresarial para la construcción de viviendas. Caso de estudio Loja-Ecuador. Ponencia, VI Congreso RIIPRO, Medellín Colombia.

[5] Cleland, D. \& King, W. (2014) Manual para la Administración de Proyectos (págs. 15-53). México: Patria.

[6] Delgado, R. (2012), La Dirección Integrada de Proyectos haciendo uso de las Nuevas Tecnologías de la Informática y las Comunicaciones. Libro de texto. Editado por CETA. ISPJAE. Cuba.

[7] Díaz, V (2014). El concepto de ciencia como sistema, el positivismo, neopositivismo y las "investigaciones cuantitativas y cualitativas" Revista Reflexiones Vol. 30, $\mathrm{N}^{\circ}$ 2, 2014 ISSN 0120-5552 http://dx.doi.org/10.14482/ sun.30.1.4309

[8] Espinet, S. \& Sánchez, M.E. (2017) Ciencia del Proyecto. Aplicación a proyectos de construcción. España: Editorial Académica Española. ISBN 978-3-639-76816-9

[9] Espinet, S. \& Sánchez, M.E. (2017) Investigación Cualitativa en Arquitectura Aplicación a proyectos de construcción. España: Editorial Académica Española. ISBN 978-3-63948757-2

[10] Espinet, S. (2007) Notas de clase: (Ciencia del Proyecto, Maestría en Construcción Civil, UNL), Loja - Ecuador.

[11] García, F. (2006). Compendio de arquitectura legal: derecho profesional y valoraciones inmobiliarias. Barcelona: Editorial Reverté.

[12] Heredia, R. (1995) Dirección Integrada de Proyecto - DIP "Project Management". 2a Edición. Escuela Técnica Superior de Ingenieros Industriales. Universidad Politécnica de Madrid.

[13] Hernández Sampieri y otros, (2016): Metodología de la Investigación. Editorial McGraw Hill. Sexta Edición. México.

[14] LLEDO, P. (2014) “Dirección Profesional de Proyectos: como aprobar el PMP", 4ra. Edición. Victoria, BC, Canadá, pp.328330. ISBN 978-987-05-5681-7.

[15] Macías, J. \& Gonzales L. (2010), Ciencia del Proyecto, Matanzas, Cuba.

[16] Palacios, C. (2003) Ejecución y Control de Obras Civiles, Ingeniería del Tercer Milenio. SEDAB, Loja Ecuador

[17] PROJECT MANAGEMENT INSTITUTE (PMI). "A guide to the project management. Body of knowledge". (PMBOK Guide), 6ta Edición. 2016.

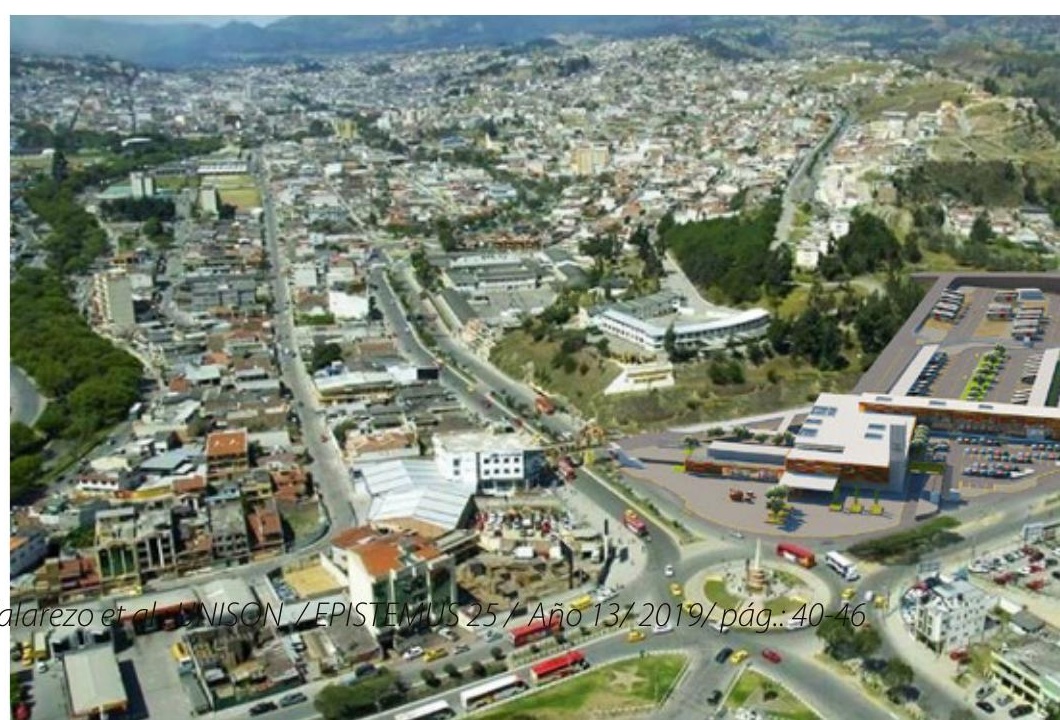

